\title{
CARACTERÍSTICAS TEMPORALES DEL LENGUAJE ORAL INFANTIL (INVESTIGACIONES COMPARATIVAS ENTRE NIÑOS ESPAÑOLES Y \\ RUSOS)
}

\author{
ABAKUMOVA, OLGA y LÓPEZ GONZÁLEZ, HERMENEGILDO1 \\ Universidad Estatal de Voronezh (Rusia) y Universidad de León
}

Hoy en día el habla infantil y sus parámetros representan un fenómeno poco estudiado aún, aunque, a pesar de muchos inconvenientes, va abriéndose camino en campos como la lingüística y la psicolingüística. El presente trabajo representa un intento de describir las características temporales del habla infantil desde el punto de vista de su ritmo, y ello a partir de una serie de grabaciones realizadas tanto en español como en ruso. Para llevar a cabo dicho análisis, hemos utilizado el método auditivo. Así, para el estudio de este fenómeno hemos elegido, como material de

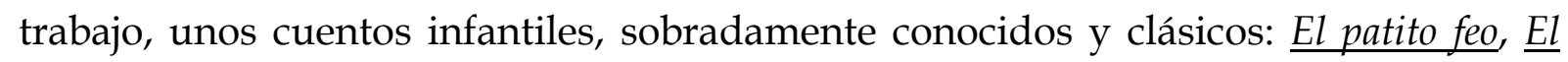
lobo y los siete cabritillos y La Cenicienta.

Como significa la conocida investigadora en el campo del habla oral, la Dra L. V. Velíchkova (Universidad Estatal de Voronezh),

el ritmo del habla como fenómeno universal del lenguaje oral se refiere al proceso de organización del material lingüístico en el habla y a los mecanismos generadores; es decir, los procesos lingüísticos y psicolingüísticos (1998: 27).

Estas páginas responden, entonces, al hecho de que es necesario investigar determinados parámetros del habla infantil, desde un aspecto comparativo, lo que

\footnotetext{
1 Profesora de la Universidad de Voronezh (Rusia). Correo-e: aba@icmail.ru; olga2000@hotbox.ru Profesor de la Universidad de León. Correo-e: hlopg@unileon.es Recibido: 12-03-2009; segunda versión: 28-04-2009.
} 
nos llevará a elevar algunas conclusiones sobre el hecho del acercamiento al aprendizaje de una lengua segunda, por lo que vamos a intentar una breve aportación al respecto.

En primer lugar, resulta procedente decir que la definición de los parámetros del habla infantil incluye también la característica de las pausas, a las que haremos posteriormente mención. Así, según A. M. Antípova

"la pausa" ha de ser entendida como una interrupción temporal. Como cualquier otro fenómeno del habla, la pausa es polifuncional. Se puede estudiar la pausa desde los siguientes puntos de vista: a) su posición en el sintagma, b) su posición en la frase, c) su longitud, d) carácter temporal, e) la actitud del hablante a la enunciación, f) la orientación del funcionamiento de la pausa.

Las pausas se dividen en finales y no finales. Se denominan finales las pausas que aparecen al terminar unidades semánticas completas. Las pausas finales se encuentran dentro de la frase y reparan un sintagma de otro. La pausa de sintagma, es decir, la pausa no final, separa un sintagma de otro y se encuentra entre palabras, con independencia de la relación semántica. La pausa dentro de un sintagma sirve para la asignación de cierto elemento de la oración. La pausa no llena es un intervalo, una interrupción en el sonido. Si la pausa se rellena de los sonidos no comunicativos, puede ser nombrada llena. Las pausas llenas igual que no llenas son características para el habla espontánea. La elección de la pausa depende de la manera individual del hablante y de la densidad emocional de la situación (Antípova, 1984: 47).

En el estudio del habla aparece un problema particular, el de la clasificación de las pausas, ya que, en el habla espontánea, la unidad principal semántica y formal es el grupo rítmico, por es necesario fijar, en dicho grupo rítmico, las pausas finales y las del sintagma, además de las propias de este último.

Conscientes de este problema previo, la estrategia de la elaboración del material experimental práctico incluyó la determinación de las pausas entre los grupos rítmicos. La definición tuvo, al mismo tiempo, el carácter de un medio auxiliar para la magnitud de los grupos rítmicos (GR).

A los "auditores expertos" se les proponía marcar las pausas en los textos de los niños (han sido registradas las pausas de cualquier tipo: pequeña $(\xi)$, mediana $(/ /)$, larga $(/))$. Este hecho nos permitió determinar los grupos rítmicos concretos 
(GR). Estos datos del habla infantil española y rusa han sido sometidos, naturalmente, a la interpretación lingüística. La imagen rítmica general de los textos infantiles, según los datos análisis discrecional de los auditores, referidos al habla infantil española y rusa, está representada en las tablas siguientes.

Característica de la magnitud de los grupos rítmicos en el habla infantil rusa

Tabla №1/r El patito feo

\begin{tabular}{|c|c|c|c|}
\hline № & Entrevistados & $\begin{array}{l}\text { La longitud media supuesta de } \\
\text { los grupos rítmicos en las sílabas }\end{array}$ & $\begin{array}{c}\text { Parámetros de la } \\
\text { longitud real de los GR }\end{array}$ \\
\hline 1 & E1/3niña/r & 9,62 & $\begin{array}{c}\text { Qmin }=2 \\
\text { Qmax }=7 \\
Q f=7 ; 4\end{array}$ \\
\hline 2 & E 1/4niño/r & 7,20 & $\begin{array}{c}\mathrm{Qmin}=1 \\
\mathrm{Qmax}=21 \\
\mathrm{Qf}=8 ; 1\end{array}$ \\
\hline 3 & E 1/5niña/r & 11 & $\begin{array}{c}\text { Qmin=1 } \\
\text { Qmax }=11 \\
Q f=5 ; 3\end{array}$ \\
\hline 4 & E 6/6niña/r & 5,33 & $\begin{array}{c}\mathrm{Qmin}=2 \\
\mathrm{Qmax}=7 \\
\mathrm{Qf}=3\end{array}$ \\
\hline 5 & ИE2/7niña/r & 5,72 & $\begin{array}{c}\text { Qmin }=1 \\
\text { Qmax }=12 \\
Q f=5 ; 4\end{array}$ \\
\hline
\end{tabular}

Tabla №2/r El lobo y los siete cabritillos

\begin{tabular}{|c|c|c|c|}
\hline № & Entrevistados & $\begin{array}{l}\text { La longitud media supuesta de } \\
\text { los grupos rítmicos en las sílabas }\end{array}$ & $\begin{array}{c}\text { Parámetros de la } \\
\text { longitud real de los GR }\end{array}$ \\
\hline 1 & E 3/3niño/r & 5 & $\begin{array}{l}\mathrm{Qmin}=3 \\
\mathrm{Qmax}=8 \\
\mathrm{Qf}=3 ; 4 ; 5\end{array}$ \\
\hline 2 & E 1/4niño/r & 5,04 & $\begin{array}{c}\text { Qmin }=1 \\
\text { Qmax }=13 \\
\text { Qf }=4 ; 6\end{array}$ \\
\hline 3 & E 9/5niño/r & 9,91 & $\begin{array}{c}\text { Qmin }=1 \\
\text { Qmax }=20 \\
\text { Qf }=10 ; 6\end{array}$ \\
\hline 4 & E 1/6niño/r & 8,13 & $\begin{array}{c}\text { Qmin }=1 \\
Q \max =11 \\
Q f=6 ; 3\end{array}$ \\
\hline 5 & E 4/7niño/r & 7,64 & $\begin{array}{c}\mathrm{Qmin}=1 \\
\mathrm{Qmax}=12 \\
\mathrm{Qf}=5 ; 4\end{array}$ \\
\hline
\end{tabular}


Tabla №3/r La Cenicienta

\begin{tabular}{|c|c|c|c|}
\hline № & Entrevistados & $\begin{array}{l}\text { La longitud media supuesta de } \\
\text { los grupos rítmicos en las sílabas }\end{array}$ & $\begin{array}{c}\text { Parámetros de la } \\
\text { longitud real de los GR }\end{array}$ \\
\hline \multirow[t]{3}{*}{1} & \multirow[t]{3}{*}{ E 8/3niño/r } & \multirow[t]{3}{*}{4,66} & $\mathrm{Qmin}=1$ \\
\hline & & & $Q \max =6$ \\
\hline & & & $Q f=3 ; 5$ \\
\hline \multirow[t]{3}{*}{2} & \multirow[t]{3}{*}{ E 2/4niña/r } & \multirow[t]{3}{*}{3,88} & Qmin $=1$ \\
\hline & & & Qmax $=7$ \\
\hline & & & $\mathrm{Qf}=1$ \\
\hline \multirow[t]{3}{*}{3} & \multirow[t]{3}{*}{ E 1/5niña/r } & \multirow[t]{3}{*}{11,25} & Qmin $=1$ \\
\hline & & & $\widehat{Q m a x}=12$ \\
\hline & & & $\mathrm{Qf}=2 ; 5$ \\
\hline \multirow[t]{3}{*}{4} & \multirow[t]{3}{*}{ E 10/6niño/r } & \multirow[t]{3}{*}{12} & $\mathrm{Qmin}=2$ \\
\hline & & & Qmax $=11$ \\
\hline & & & $\mathrm{Qf}=2 ; 3$ \\
\hline \multirow[t]{3}{*}{5} & \multirow[t]{3}{*}{ E 5/7niño/r } & \multirow[t]{3}{*}{6,09} & Qmin $=1$ \\
\hline & & & Qmax $=12$ \\
\hline & & & $Q f=3$ \\
\hline
\end{tabular}

Los datos obtenidos permiten hablar de la magnitud de los grupos rítmicos concretos. Según el cuento infantil El patito feo el grupo de longitud mínima (Qmin=1) ha sido representado, en el habla de los niños/as de 3, 4, 5, 7 años, del modo siguiente:

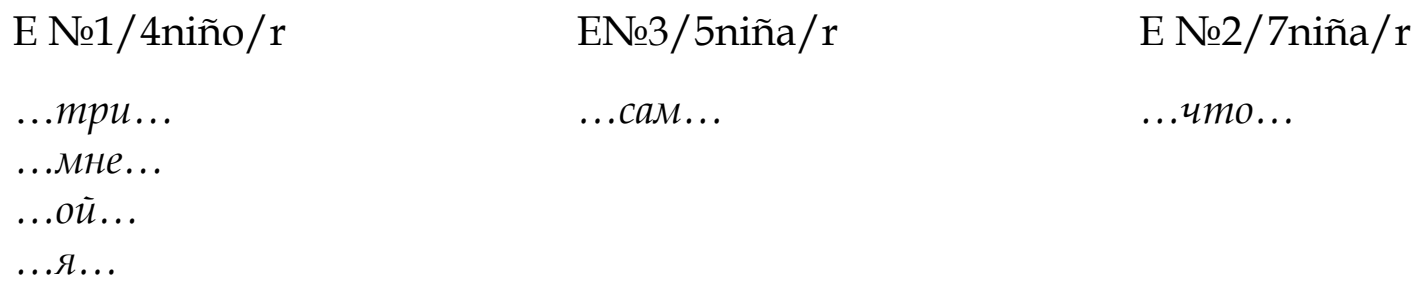

El grupo de longitud máxima (Qmax=21, 11, 12), en el habla infantil de los niños/as de entre 4 y 7 años, tiene las siguientes realizaciones:

...эт четыле утенка плавают а эта один утенок плавает...

...от собаки уплывает а то она его съест и его не будет...

GR Qmax=21 es ocasional, ya que no hay pausas en el habla.

E №1/5niña/r, GR Qmax=11

...ну вот как в сказке он говорил так и ... 
E №2/7niña/r, GR Qmax=12

...я не хочу вам зла я просто а щас...

Según los cálculos, los grupos rítmicos de frecuencia (Qf) son:

$\mathrm{A} \operatorname{los} 4$ años $\mathrm{Qf}=8$

E №1/4niño/r

...ну потому что нлавится...

...мне залко чтобы собака...

...утенка съела залко мне...

А как она включается?

Уменя уже прошла Все

E №1/3niña/r, donde $\mathrm{Qf}=7$

Мне он не понравился.

За то что он хотел...

Заклевали шоб его...

Это гадкий утенок.

...когда он стал лебеденком...

E №1/5niña/r y E №2/7niña/r demostraron $Q f=5$

E №1/5niña/r $\quad$ E №2/7niña/r

...немог убежать Не бейте меня

Убейте меня

По-гусиному

...поклюйте меня

...меня совсем...

...никто не ююбит

E №1/3niña/r y E №2/7niña/r tienen $Q f=4$

E №1/3niña/r $\quad$ E №2/7niña/r

А почему? ...mо плохое...

...понравился... ..щцас вот эта...

Красивым... ..склюйте меня...

...утки клюют...

Cabe mencionar que los niños de 4 y 7 años tienen el mismo Qf y la misma longitud media supuesta de los grupos rítmicos, en las sílabas, hecho que nos permite hablar de la homogeneidad de la magnitud de los grupos rítmicos. Por el contrario, en los niños de 3, 5 y 6 años, de acuerdo con los datos distintos, por la longitud media de los grupos rítmicos, obtenemos GR heterogéneos por su magnitud Qf. 
De acuerdo con las conclusiones obtenidas del cuento infantil El lobo y los siete cabritillos, los entrevistados (E) №1/4niño/r, E №9/5niña/r, E № 1/6niño/r, E №4/7niño/r tienen un Qmin=1 que se presenta en los textos del modo siguiente:

$\begin{array}{llll}\text { E №1/4niño/r, } & \text { E №9/5niña/r, } & \text { E №1/6niña/r, } & \text { E №4/7niño/r } \\ \text {...вот... } & \text { Hy... } & \ldots \text {.... } & \ldots 3 a \ldots\end{array}$

Los E №9/5niña/r, E №1/6niño/r tienen Qmax=20, que aparece de esta manera:

\section{E №9/5niña/r}

Ваша мать пришла молочка принесла бежит молочко по вымечку

E №1/6niño/r

...всех козлят а один схоронился он его не увидел и ушел...

El grupo rítmico de frecuencia (Qf), en el mismo cuento, tiene las siguientes realizaciones:

E №1/4niño/r

...козленок один был...

...один остался он...

...это я ваша мать.

...молока привезла...

E №1/6niño/r

...короче там ямку...

...стали они прыгать...

...яму с горящим и ...

...молочко принесла.
E №9/5niña/r

Молочка принесла...

...из копытечку...

E №4/7niño/r

Семь волков бежали...

А волки спрятались...

...nобежали за ним...

И волки подрались...

El Qf=4 aparece, en el E №3/3niño/r, de este modo:

Не знаю.

Белые.

He xочy.

La niña de 5 años, E №9/5niña/r tiene un Qf=10

...на части раз... эта разорвали...

Ombopumecя omonрuтеся. 


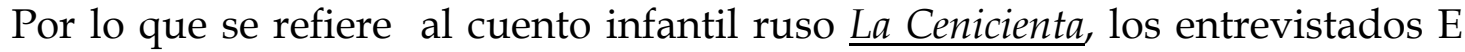
№8/3niño/r, E №3/4niña/r, E №1/5niña/r y E №5/7niño/r tienen un Qmin=1, de este modo:
.......
To...
...bom...
... мышь...
Hy...
Зльм.

Por el contrario, los E №10/6niño/r y E №5/7niña/r, muestran un Qmax=12, 11:

E №10/6niño/r

... и лохмотьях и прини не узнал бы ее.

E №5/7niña/r

...карета ее превратилась в тыкву...

Qf aparece en el habla infantil, del modo siguiente:

$\mathrm{Qf}=3$ poseen, en este caso, los E №8/3niño/r y E №5/7niño/r

$\begin{array}{ll}\text { Е №8/3niño/r } & \text { Е №5/7niño/r } \\ \text { Забыла... } & \text { О время... } \\ \ldots \text {.что она... } & \text { Весело... } \\ \text { А еще.... } & \text { Весело... } \\ \ldots \text {..есело... } & \\ \text { Сестрицы. } & \end{array}$

E №1/5niña/r y E №10/6niño/r exhiben un Qf = 2:

E №1/5niña/r $\quad$ E №10/6niño/r

То бы... Тогда б...

Грустным. Грубым.

Грубым.

Según los datos recogidos con el cuento infantil El lobo y los siete cabritillos, los niños de 6 y 7 años no tienen la misma longitud de los GR en sílabas y Qf. El cuento infantil $\underline{\mathrm{La} \text { Cenicienta }}$ nos ha permitido observar que la longitud de los GR, en media supuesta, tampoco concuerda en todos los grupos de edad, lo que significa que los grupos rítmicos no son homogéneos. El hecho se debe al uso de los clichés de los cuentos infantiles, que representan cierta dificultad lingüística (por ejemplo, козлятушки, ребятушки). 
La relación entre los GR de frecuencia y el estado emocional del niño hablante se observa de este modo:

1. los grupos rítmicos de frecuencia con mayor longitud $(\mathrm{Qf}=8,7,5)$ aparecen como expresión de las emociones negativas.

2. Los grupos rítmicos de frecuencia menor $(\mathrm{Qf}=3,4)$ aparecen, por el contrario, en las emociones positivas.

El material recogido, que representa los grupos de frecuencia después del análisis sintáctico, incluye la información sobre los tipos de sintagmas de frecuencia en el habla infantil de acuerdo con la edad. En este material se observa claramente el conjunto de los modelos sintácticos (ver más arriba nuestras conclusiones sobre el cuento infantil El Patito feo ENo1/3niña/r, donde Qf = 7; E №2/7niña/r, donde Qf = 4; el cuento infantil El lobo y siete cabritillos E №1/4niño/r y E №1/6niño/r, donde Qf $=6$ y E №3/3niño/r, donde Qf = 3; o, para terminar, los datos aportados por el cuento infantil La Cenicienta E №1/5niña/r, donde Qf = 2)

\section{Característica de la magnitud de los grupos rítmicos en el habla infantil española}

Tabla №1/esp El patito feo

\begin{tabular}{|c|c|c|c|}
\hline № & Entrevistados & $\begin{array}{l}\text { La longitud media supuesta de los } \\
\text { grupos rítmicos en las sílabas }\end{array}$ & $\begin{array}{c}\text { Parámetros de la } \\
\text { longitud real de los GR }\end{array}$ \\
\hline 1 & $\begin{array}{c}\text { E } \\
\text { 3/3niño/esp }\end{array}$ & 5,06 & $\begin{array}{c}\text { Qmin }=1 \\
Q \max =11 \\
Q f=2,5\end{array}$ \\
\hline 2 & $\begin{array}{c}\mathrm{E} \\
\text { 4/4niña/esp }\end{array}$ & 4,70 & $\begin{array}{c}Q \min =2 \\
Q \max =8 \\
Q f=2,4\end{array}$ \\
\hline 3 & $\begin{array}{c}\mathrm{E} \\
5 / 5 \text { niño/esp }\end{array}$ & 4,33 & $\begin{array}{l}\mathrm{Qmin}=1 \\
\mathrm{Qmax}=8 \\
\mathrm{Qf}=23+\end{array}$ \\
\hline 4 & $\begin{array}{c}\mathrm{E} \\
\text { 5/6niña/esp }\end{array}$ & 6,42 & $\begin{array}{c}\mathrm{Qmin}=1 \\
\mathrm{Qmax}=8 \\
\mathrm{Qf}=2,7 \text { э+ э- }\end{array}$ \\
\hline 5 & $\begin{array}{c}\mathrm{E} \\
\text { 5/7niña/esp }\end{array}$ & 4,16 & $\begin{array}{c}\mathrm{Qmin}=1 \\
\mathrm{Qmax}=5 \\
\mathrm{Qf}=2,5\end{array}$ \\
\hline
\end{tabular}


Tabla №2/esp El lobo y los siete cabritillos

\begin{tabular}{|c|c|c|c|}
\hline № & Entrevistados & $\begin{array}{c}\text { La longitud media supuesta de los } \\
\text { grupos rítmicos en las sílabas }\end{array}$ & $\begin{array}{c}\text { Parámetros de la } \\
\text { longitud real de los GR }\end{array}$ \\
\hline 1 & $\begin{array}{c}\text { E } \\
\text { 3/3niño/esp }\end{array}$ & 6,33 & $\begin{array}{c}\text { Qmin }=1 \\
Q \max =6 \\
\mathrm{Qf}=1 ; 2 ; 3 ; 5 ; 6\end{array}$ \\
\hline 2 & $\begin{array}{c}\text { E 3/4 } \\
\text { niña/esp }\end{array}$ & 6,28 & $\begin{array}{c}\mathrm{Qmin}=1 \\
\mathrm{Qmax}=10 \\
\mathrm{Qf}=3\end{array}$ \\
\hline 3 & $\begin{array}{c}\text { E 1/5 } \\
\text { niño/esp }\end{array}$ & 10,75 & $\begin{array}{c}\text { Qmin=1 } \\
Q \max =11 \\
Q f=5\end{array}$ \\
\hline 4 & $\begin{array}{c}\text { E } 4 / 6 \\
\text { niño/esp }\end{array}$ & 4,70 & $\begin{array}{c}\mathrm{Qmin}=1 \\
\mathrm{Qmax}=7 \\
\mathrm{Qf}=3\end{array}$ \\
\hline 5 & $\begin{array}{c}\text { E } 2 / 7 \\
\text { niño/esp }\end{array}$ & 5,33 & $\begin{array}{c}\mathrm{Qmin}=1 \\
\mathrm{Qmax}=12 \\
\mathrm{Qf}=2\end{array}$ \\
\hline
\end{tabular}

Tabla №3/esp La cenicienta

\begin{tabular}{|c|c|c|c|}
\hline № & Entrevistados & $\begin{array}{l}\text { La longitud media supuesta de los } \\
\text { grupos rítmicos en las sílabas }\end{array}$ & $\begin{array}{c}\text { Parámetros de la longitud } \\
\text { real de los GR }\end{array}$ \\
\hline 1 & E 2/3 niño/esp & 6,50 & $\begin{array}{c}\text { Qmin }=1 \\
\text { Qmax }=25 \\
\text { Qf }=2\end{array}$ \\
\hline 2 & E 2/4 niño/esp & 7,25 & $\begin{array}{c}\text { Qmin=2 } \\
\text { Qmax }=80 \\
Q f=2\end{array}$ \\
\hline 3 & E $4 / 5$ niña/esp & 3,25 & $\begin{array}{c}\mathrm{Qmin}=1 \\
\mathrm{Qmax}=8 \\
\mathrm{Qf}=2 ; 1\end{array}$ \\
\hline 4 & E 3/6 niña/esp & 3,83 & $\begin{array}{c}\mathrm{Qmin}=2 \\
\mathrm{Qmax}=6 \\
\mathrm{Qf}=2\end{array}$ \\
\hline 5 & E 5/7 niño/esp & 7,00 & $\begin{array}{c}\text { Qmin }=1 \\
\text { Qmax }=12 \\
\text { Qf }=2 ; 3\end{array}$ \\
\hline
\end{tabular}

Los datos recogidos por medio del cuento infantil El patito feo nos indican la magnitud de los grupos rítmicos Qmin $=1$ entre los 3 y 7 años. Se encuentran en el habla del modo siguiente: 


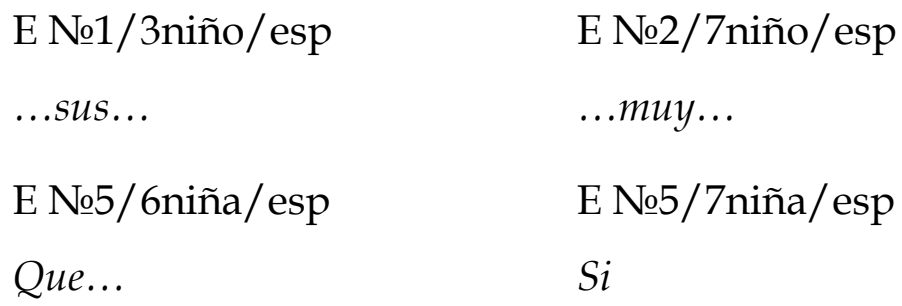

Únicamente el entrevistado, E №1/3niño/esp, tiene un Qmax=11:

El patito feo si que hablaba

El sujeto entrevistado, E №4/4niña/esp, muestra un GR Qmin =2; ello nos demuestra que, curiosamente, en este caso, Qmin coincide con Qf.

De que...

Que se...

Muy muy...

...fuerte

Hemos encontrado el caso de una niña, E №4/4niña/esp, donde el GR Qmax=8:

Porque me alegra mucho

Los grupos rítmicos, donde $\mathrm{Qf}=2$, se observan en todos los grupos de edad y están representados del modo siguiente:

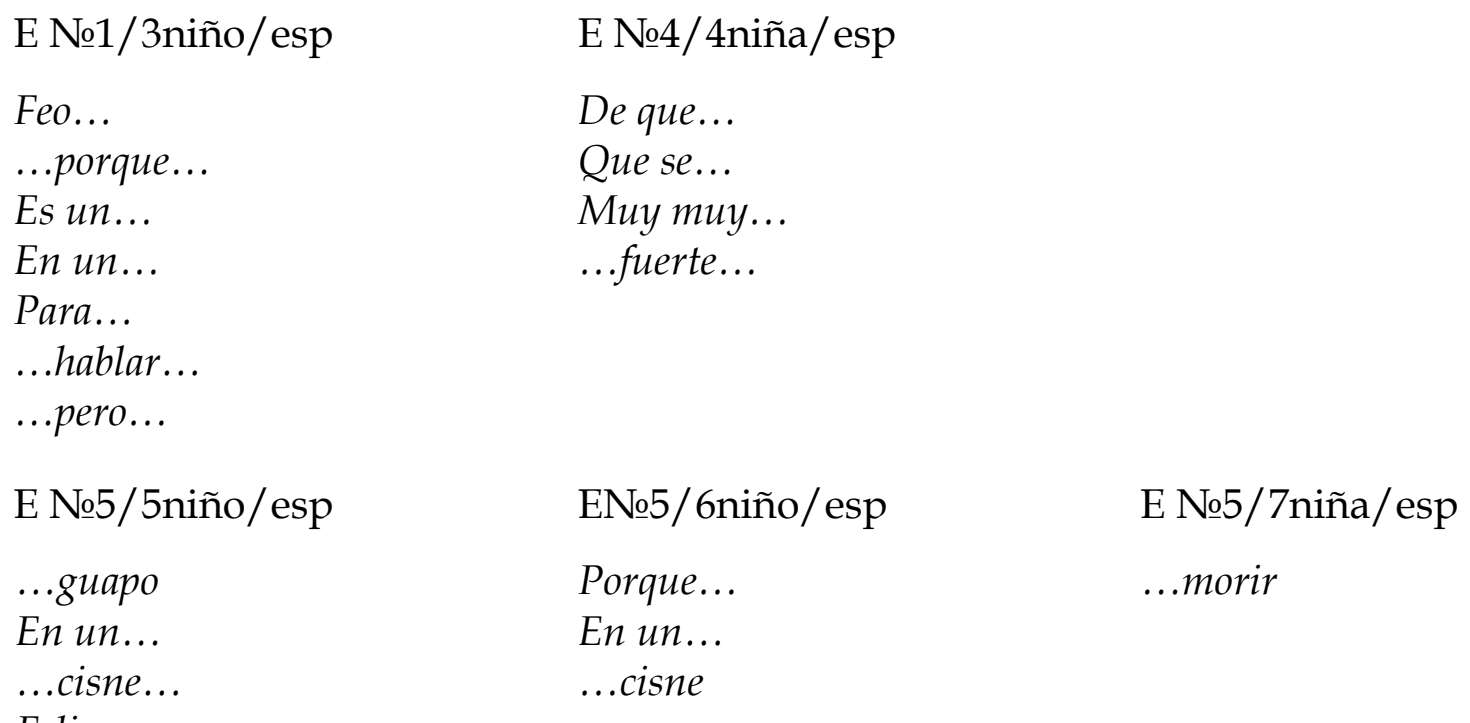

\section{Feliz}

Los grupos rítmicos Qmax =8 aparecen en los niños/as de 4, 5 o 6 años:

E №4/4niña/esp

Porque me alegra mucho 
E №5/5niño/esp

Soy feliz porque me quieren

E №5/6niño/esp

...cuando era pequeñito...

Los niños de 7 años muestran una equivalencia entre los Qmax y Qf.

E №5/6niña/esp tiene GR Qmax=5

...se convirtió en...

...feliz y alegre

Es importante señalar que los grupos rítmicos Qf y la longitud media supuesta de los grupos rítmicos coinciden a $\operatorname{los} 3,4,6$ y 7 años, los que indica la homogeneidad de los grupos rítmicos, y, únicamente a los 5 años, la magnitud Qf y la de los grupos rítmicos no concuerdan.

Por lo observado en el cuento El lobo y siete cabritillos, Qmin $=1$ aparece en todos los grupos de edad, o sea de los 3 a los 7 años.

$\begin{array}{lll}\text { E №3/3niño/esp } & \text { E№3/4niña/esp } & \text { E №1/5niño/esp } \\ \text { El } & \text { Muy... } & \text { Pues... } \\ \text {...lo } & \text { Muy... } & \\ \text { E №4/6niño/esp } & \text { E №2/7niño/esp } \\ \text { Que... } & \text { Que... } & \\ & \text { Soy... }\end{array}$

Qmax=6 aparece en el entrevistado E №3/3niño/esp

Se corta al lobo...

La tenía ronca...

Qmax=7 exhibe el sujeto E №4/6niño/esp

...el lobo quería ser...

Qmax=10 muestra el E №3/4niña/esp

...la mamá Cabra tenía que...

Qmax=11 presenta el E №1/5niño/esp

Abridme la puerta, soy vuestra mamá 
Qmax=12 tiene ya un niño identificado como E №2/7niño/esp

...al cabrito se lo hubiera comido

Los Qf están representados en el habla infantil del modo siguiente:

A $\operatorname{los} 3,4,6$ años:

$\mathrm{Qf}=3$

E №3/3niño/esp

ENo3/4niña/esp

E №4/6niño/esp

El lobo...

ellobo...

...las como

...se come...

...espera...

Muy fuerte

... y los ca...

...britos los...

Muy suave

Abrid...

E №1/5niño/esp, donde Qf=5:

Los cabritillos

Llegaros aquí

Of=2 en el sujeto E №2/7niño/esp:

Por que...

Suave

Fuerte...

...ronco

La magnitud de los grupos rítmicos Qmin =1 a los 3, 5 y 7 años, según el cuento de La Cenicienta está representada así:
E №2/3niño/esp
ENo4/5niña/esp
E №5/7niño/esp
...las...
Que...
Muy...
...el...
...que...

Hay un entrevistado, E №2/3niño/esp que tiene un GR Qmax=25

Que se olvidará en su casa su zapatito y su zapato

Los grupos rítmicos Qmax=25 son ocasionales ya que no aparecen pausas en el habla.

Encontramos también grupos rítmicos $Q \max =6,8,11,12$, en los niños de entre 4 y 7 años, y han sido reproducidos del modo siguiente: 
E №2/3niño/esp, donde Qmax=8

...acordaría de nada.

E №4/5niña/esp, donde Qmax=8

Y lleva la diadema...

E №3/6niña/esp, donde Qmax=6

Porque eran malas.

E №5/7niño/esp, donde Qmax=12

Porque todo el día estaba gritando...

Los grupos rítmicos con Qf=2, según los datos del cuento infantil La Cenicienta, así como los recogidos en sus formas de narrar El patito feo, aparecen en todos los grupos de edad y vienen a ser de este modo:

$\begin{array}{lll}\text { E №2/3niño/esp } & \text { ENo2/4niño/esp } & \text { E №4/5niña/esp } \\ \text { Feliz... } & \text { Baja } & \text { Alta } \\ \text { Quela... } & \text { Altas } & \text { Bajas } \\ \text { Malas } & \text { Feas } & \\ & \text { Porque... } & \\ & \ldots \text {..eran ... } & \\ & \ldots . \text { malas... } & \\ \text { E №3/6niña/esp } & \text { E №5/7niño/esp } & \\ \text { Que se... } & \text { Que se... } & \\ \text { Buena } & \ldots . \text { suave } & \\ \text { Triste } & \text { Porque... }\end{array}$

Los de edades diferentes no muestran, por lo constatado, la misma longitud media supuesta de los grupos rítmicos en sílabas y Qf; es decir los grupos rítmicos, por ellos utilizados, son del todo heterogéneos.

Así, la magnitud de los grupos rítmicos Qmin=1 viene determinada por los siguientes factores lingüísticos: la presencia tanto en el habla infantil rusa, como en la española, de las palabras monosilábicas con mayor frecuencia que el resto y que pueden ser enmarcadas en el primer grupo rítmico, por ejemplo, ...я...; ...сам...; ..иmо.../..que...; ...si...; ...muy.... 
La magnitud de los grupos rítmicos Qmax=7, 8 está presente, en sus máximos, en los niños rusos a la edad des 3 y 4 años; a los 5, 6, 7, años aparecen ya parámetros de Qmax=11,12.

En el habla infantil española Qmax aparece del modo siguiente: a los 3 años Qmax=6, 11, a los 4, 5, 6 años Qmax=8 y a los 7 años Qmax=5, 12.

Por lo que hace a la magnitud de los grupos rítmicos $Q f=3$, 5, la misma aparece en todos los grupos de edad de ambas nacionalidades y en ambas lenguas; sirvan como ejemplo:

Убейте меня...,...никто не любит...,....se convirtió еп ...,...feliz y alegre...

\section{Abreviaturas}

1. grupos rítmicos - GR

2. entrevisado - E

3. ruso $-r$

4. español - esp

5. E 8/3niño/r - entrevistado №8 de 3 años, niño ruso

6. E 2/4niña/r - entrevistado №2 de 4 años. niña rusa

7. E №3/6niña/esp - entrevistado № 3 de 6 años, niña española

8. E №5/7niño/esp - entrevistado № 5 de 7 años, niño español

9. Qmax - grupo rítmico de longitud máxima

10. Qmin - grupo rítmico de longitud mínima

11. Qf - grupo rítmico de frecuencia 


\section{BIBLIOGRAFÍA}

Abakumova, O. y López González, H. (2008): “Descripción de la percepción del habla emocional infantil: teoría y práctica", Estudios humanísticos. Filología, 30: 351-360, León.

Antípova, A.P. (1984): Sistema del ritmo del habla inglesa, Moscú.

Velíchkova, L.V. (1998): Enfoque contrastivo en la investigación del ritmo del habla, Voronezh.

Izard, C. E. (1977): Human emotions, New York. 